\title{
Cirurgia bariátrica: Acurácia perceptiva, satisfação corporal e qualidade de vida
}

\author{
Bariatric surgery: Perceptual accuracy, body satisfaction and quality of life \\ Cirugía bariátrica: Precisión perceptiva, satisfacción corporal y calidad de vida
}

Recebido: 14/07/2021 | Revisado: 19/07/2021 | Aceito: 22/07/2021 | Publicado: 29/07/2021

\author{
Gladson Henrique Silva \\ ORCID: https://orcid.org/0000-0002-7572-4076 \\ Universidade Federal de São João del Rei, Brasil \\ E-mail: gladsonhenrique150@gmail.com \\ Ricardo Bezerra Cavalcante \\ ORCID: https://orcid.org/0000-0001-5381-4815 \\ Universidade Federal de Juiz de Fora, Brasil \\ E-mail: ricardocavalcante.ufjf@gmail.com \\ Sandra Valenzuela Suazo \\ ORCID: https://orcid.org/0000-0002-1308-4835 \\ Universidad de Concepción, Chile \\ E-mail: sandra.valenzuela.suazo@gmail.com \\ Bernardo Guimarães de Aguiar \\ ORCID: https://orcid.org/0000-0001-7030-1165 \\ Universidade Federal de São João del Rei, Brasil \\ E-mail: baguiar40@gmail.com \\ Samuel Barroso Rodrigues \\ ORCID: https://orcid.org/0000-0002-9832-5510 \\ Universidade de Itaúna, Brasil \\ E-mail: samuelbarroso88@gmail.com \\ Richardson Miranda Machado \\ ORCID: https://orcid.org/0000-0001-9895-6905 \\ Universidade Federal de São João del Rei, Brasil \\ E-mail: richardson@ufsj.edu.br
}

\section{Resumo}

A Organização Mundial de Saúde aponta a obesidade como um dos maiores problemas de saúde pública. Quando os tratamentos convencionais, baseados em dietas com a restrição da ingestão calórica e prática de atividades físicas não mostraram resultados satisfatórios, nos casos mais graves, tornou-se indicativo a realização da cirurgia bariátrica. Sendo assim, o trabalho se faz relevante, tendo em vista a busca pela elucidação da relação que o paciente tem com seu corpo, quanto ao aspecto perceptual, bem como à sua qualidade de vida. Nesse sentido, este estudo teve como objetivo avaliar a acurácia perceptiva, a satisfação corporal e a qualidade de vida dos pacientes submetidos à cirurgia bariátrica. Trata-se de estudo transversal, realizado com 305 pacientes de uma clínica de médio porte, referência na execução de cirurgias bariátricas na região Centro-Oeste de Minas Gerais. Foi utilizada uma escala de avaliação da acurácia perceptiva e satisfação corporal e outra para avaliar a qualidade de vida. Realizou-se análise descritiva e de associação dos dados. O estudo demonstrou que houve distorção da imagem corporal e insatisfação dos pacientes quanto a seus corpos no período estudado. A qualidade de vida foi considerada boa em todos os domínios. Não houve influência do tempo pós-cirurgia nos resultados. Então pode-se afirmar que a cirurgia bariátrica é eficaz, quanto à qualidade de vida, contudo a acuraria perceptiva e satisfação com a imagem corporal demonstram merecer maior atenção em razão dos resultados encontrados.

Palavras-chave: Enfermagem psiquiátrica; Cirurgia bariátrica; Imagem corporal; Qualidade de vida.

\begin{abstract}
The World Health Organization points to obesity as one of the biggest public health problems. When the conventional treatments based on diets with the restriction of caloric intake and practice of physical activities have not shown satisfactory results, in the most severe cases, bariatric surgery becomes indicative. Therefore, the work is relevant in view of the search for elucidating the relationship that the patient has with its body concerning the perceptual aspect as well as its quality of life. In this sense, this work aimed to evaluate the perceptual accuracy, body satisfaction and quality of patients' life undergoing bariatric surgery. This is a cross-sectional study carried out with 305 patients from a medium-sized reference clinic in the execution of bariatric surgeries in the Midwest region of Minas Gerais. One scale was used to assess perceptual accuracy and body satisfaction and another one to assess quality of life. Descriptive and association analysis of data were performed. The study showed that there was distortion of body's image and patients' dissatisfaction with their bodies in the studied period. Quality of life was considered good in all areas. There was no time influence after surgery on the results. So, it can be said that bariatric surgery is effective in terms of quality of life, however the perceptive accuracy and satisfaction with body image demonstrate that it deserves greater attention due to the results found.
\end{abstract}

Keywords: Psychiatric nursing; Bariatric surgery; Body image; Quality of life. 


\section{Resumen}

La Organización Mundial de la Salud señala la obesidad como uno de nuestros mayores problemas de salud pública. Cuando los tratamientos convencionales basados en dietas con restricción del aporte calórico y la práctica de actividades físicas no han mostrado resultados satisfactorios en los casos más graves, la realización de cirugía bariátrica se vuelve indicativa. Así, el trabajo es relevante teniendo en cuenta la búsqueda de la elucidación de la relación que el paciente tiene con su cuerpo en cuanto al aspecto perceptual, así como su calidad de vida. En este sentido, este estudio pretendió evaluar la precisión perceptiva, la satisfacción corporal y la calidad de vida de los pacientes sometidos a cirugía bariátrica. Se trata de estudio transversal efectuado con 305 pacientes de una clínica de tamaño medio que es referencia en la ejecución de cirugías bariátricas en la región Centro-Oeste de Minas Gerais. Se utilizó una escala para evaluar la precisión de la percepción y la satisfacción corporal y otra para evaluar la calidad de vida. Se llevó a cabo un análisis descriptivo y de asociación de los datos. El estudio demostró que hubo distorsión de la imagen corporal e insatisfacción de los pacientes con respecto a sus cuerpos en el período estudiado. La calidad de vida se consideró buena en todos los ámbitos. No hubo influencia del tiempo posquirúrgico en los resultados. Por lo tanto, se puede decir que la cirugía bariátrica es efectiva en cuanto a la calidad de vida; sin embargo, la precisión perceptiva y la satisfacción con la imagen corporal demuestran que merecen más atención debido a los resultados obtenidos.

Palabras clave: Enfermería psiquiátrica; Cirugía bariátrica; Imagen corporal; Calidad de vida.

\section{Introdução}

A obesidade é uma doença grave e pode ocasionar diversas comorbidades de impacto para a saúde pública, como distúrbios cardiovasculares; endócrinos; respiratórios; gastrointestinais e músculos-esqueléticos. Além disso, pode atingir o sistema reprodutor e causar distúrbios psiquiátricos e outras complicações como neoplasias e a diminuição da mobilidade física (Flegal, Kit, \& Graubard, 2014; Castro, 2017; Andrade et al., 2017).

O grau de obesidade pode ser mensurado pelo Índice de Massa Corporal (IMC) e, para ser classificado como obeso, o indivíduo deve estar com índice acima de $30 \mathrm{~kg} / \mathrm{m}^{2}$. Sua gravidade pode ser classificada em grau I (moderado excesso de peso), quando o IMC se situa entre 30 e $34,9 \mathrm{~kg} / \mathrm{m}^{2}$, grau II (obesidade leve ou moderada) com IMC entre 35 e $39,9 \mathrm{~kg} / \mathrm{m}^{2}$ e, por fim, obesidade grau III (obesidade mórbida) na qual o IMC ultrapassa $40 \mathrm{~kg} / \mathrm{m}^{2}$ (Flegal et al., 2014; OMS, 2015).

Embora sua etiologia seja complexa e multifatorial, pode-se afirmar que a obesidade é o resultado da interação de diferentes fatores genéticos; metabólicos; ambientais (modernização das cidades. Soma-se a isso a concentração das populações no meio urbano); comportamentais; emocionais; doenças predisponentes e tratamentos medicamentosos (Malta et al., 2014; Francisco \& Diez-Garcia, 2015).

Dados apontam que 55,7\% da população brasileira tem excesso de peso (Brasil, 2018). O aumento da prevalência foi maior entre as faixas etárias de 18 a 24 anos (período 2006-2018). A Organização Mundial de Saúde (OMS, 2015) destaca a obesidade como um dos maiores problemas de saúde pública do mundo, com mais de 2,2 bilhões de pessoas, entre crianças e adultos, equivalente a $30 \%$ da população.

A cirurgia bariátrica é indicada para pacientes com IMC maior que $35 \mathrm{Kg} / \mathrm{m}^{2}$, grau II (obesidade leve ou moderada) ou com IMC maior que $40 \mathrm{Kg} / \mathrm{m}^{2}$, grau III (obesidade mórbida). Além do IMC, consideram-se, como propensos à cirurgia, os pacientes com comorbidades, como hipertensão arterial (HAS) e Diabetes Mellitus tipo II (DM). Os resultados assim esperados com a cirurgia bariátrica incluem: perda de peso, melhora das comorbidades relacionadas e da qualidade de vida (Wu et al., 2014; Steyer, Oliveira, Gouvêa, Echer, \& Lucena, 2016).

Neste sentido, apesar das diversas mudanças no peso e nos contornos corporais, a cirurgia bariátrica não garante necessariamente uma qualidade de vida adequada, uma vez que a redução de peso requer não apenas uma questão meramente estética, mas uma mudança de padrões alimentares, os quais, nem sempre são adotados de forma repentina e na mesma velocidade que as mudanças físicas advindas do procedimento cirúrgico. Esse processo pode precipitar o indivíduo, inclusive, a uma baixa adesão aos novos hábitos necessários, ocasionando consequências negativas, como reganho de peso, carências nutricionais e até mesmo o risco de suicídio (Steyer et al., 2016; Lacerda et al., 2018). 
Nesse cenário, a acurácia perceptiva, ou seja, a forma como o paciente percebe seu corpo, incluindo a sua altura, forma e peso e a satisfação com a imagem corporal são fundamentais para a autoaceitação e o sucesso do tratamento da obesidade por meio da cirurgia bariátrica. Diante disso, torna-se necessário conhecer melhor de que maneira os pacientes submetidos à cirurgia bariátrica lidam com sua autopercepção, a autoimagem, como também com a sua qualidade de vida. Acredita-se que os resultados possam contribuir à produção de conhecimentos que permitirão melhor atuação do trabalho dos profissionais de saúde e do cuidado aos pacientes, quanto às suas expectativas, em relação a esse procedimento. Nessa perspectiva, este estudo teve como objetivo avaliar a acurácia perceptiva, a satisfação corporal e a qualidade de vida dos pacientes submetidos à cirurgia bariátrica.

\section{Metodologia}

Trata-se de um estudo transversal, de caráter exploratório-analítico de abordagem quantitativa (Estrela, 2018). O estudo foi desenvolvido, em uma clínica de médio porte, referência na execução de cirurgias bariátricas para os 56 municípios da região Centro-Oeste de Minas Gerais. Possui uma clientela advinda de planos de saúde e atendimentos particulares.

A população do estudo foi composta por todos os pacientes atendidos pela clínica que foram submetidos à cirurgia bariátrica, entre março de 2004 e março de 2019, totalizando 15 anos de atendimentos e 1416 pacientes. O cálculo amostral foi realizado, a partir desse total de pacientes atendidos, para o qual considerou-se uma proporção de $50 \%$ para determinada característica (por se tratar de um projeto com múltiplos desfechos), valor esse que forneceu o maior tamanho amostral, para população finita (n=1416), fixando o nível de significância em 5\% (alfa ou erro tipo I) e o erro amostral em 5\%. Ao final, resultou em um "n" mínimo estimado de 303 pacientes.

A primeira etapa da coleta de dados ocorreu, a partir dos registros dos atendimentos dos pacientes, em formato físico (prontuários), disponíveis no arquivo médico estatístico da clínica supracitada. Os critérios de inclusão foram pacientes de ambos os sexos, idade igual ou maior a dezoito anos. Critérios de Exclusão: apresentar qualquer condição que interferisse na coleta e na mensuração dos dados, informações errôneas e/ou incompletas. À medida em que as informações foram extraídas dos prontuários, elas foram inseridas em um banco de dados eletrônico, criado no Statistical Package for the Social Sciences (SPSS), versão 21.0 e categorizadas em variáveis nominais e ordinais.

A segunda etapa da coleta ocorreu, por meio da aplicação de duas escalas de medida: a primeira para avaliar a acurácia perceptiva e a satisfação dos pacientes com seus corpos e a segunda para estimar a qualidade de vida dos pacientes. As referidas escalas e o Termo de Consentimento Livre e Esclarecido (TCLE) foram diagramados e disponibilizados em formato eletrônico, por meio do Google Forms, um aplicativo gratuito de administração de pesquisas que permite o envio dos formulários, por meio de link via WhatsApp e e-mail. Desse modo, os pacientes foram contatados por telefone e, ao aceitarem participar da segunda etapa da pesquisa, receberam o link para acesso online ao TCLE e às escalas, os quais responderam a partir da orientação feita previamente pelo pesquisador via telefone. É importe ressaltar que ambas as escalas podem ser autoaplicáveis.

Para avaliar a acurácia perceptiva e a satisfação com a imagem corporal, foi utilizada a Escala de figura de silhuetas. Este instrumento consiste de um conjunto de dezoito imagens (nove desenhos de silhuetas femininas e nove masculinas), que representam figuras humanas com nove variações progressivas na escala de medida, da figura mais magra à mais larga, com Índice de Massa Corporal médio: Figura 1 (IMC 17,5); Figura 2 (IMC 20,0); Figura 3 (IMC 22,5); Figura 4 (IMC 25,0); Figura 5 (IMC 27,5); Figura 6 (IMC 30,0); Figura 7 (IMC 32,5); Figura 8 (IMC 35,0) e Figura 9 (37,5) (Kakeshita \& Almeida, 2006).

Foram apresentados aos pacientes dois conjuntos de figuras, um de figuras femininas e um de figuras masculinas, ambos de 1 a 9, em ordem ascendente (da menor para a maior figura). O paciente foi assim solicitado a escolher: $1^{\circ}$ ) a figura que representasse a silhueta de seu corpo atual; $2^{\circ}$ ) a figura que representasse a silhueta de um corpo que gostaria de ter; $3^{\circ}$ ) a 
figura que representasse a silhueta de um corpo que o paciente acreditava que iria alcançar após a cirurgia bariátrica. O avaliador isentou-se de opinião na escolha das silhuetas.

A acurácia perceptiva e a satisfação com a imagem corporal foram assim calculadas: subtraindo-se o IMC da figura escolhida como sendo o seu corpo atual do IMC da figura escolhida como sendo o corpo desejado. O resultado igual a zero indicou acurácia perceptiva real e satisfação com a imagem corporal; o resultado com valor positivo indicou superestimação do tamanho corporal e o resultado com valor negativo indicou subestimação do tamanho corporal.

Em seguida, foi aplicada a Escala de Avaliação da Qualidade de Vida (World Health Organization Quality of Life Assessment-Bref, WHOQOL-bref), composta por 26 questões, sendo duas de caráter geral, que avaliaram a percepção da qualidade de vida e a satisfação com a saúde pelo paciente e outras 24 questões distribuídas em quatro domínios (Físico, Psicológico, Relações Sociais e Meio Ambiente). Cada domínio teve seu escore calculado individualmente, o que permitiu a avaliação do nível de qualidade de vida em cada contexto. Desse modo, quanto mais alto o escore melhor a qualidade de vida em cada domínio. A pontuação foi obtida por meio de uma escala do tipo Likert de cinco pontos (1 a 5). O presente estudo adaptou a classificação, agrupando os pacientes, de acordo com três classes de distribuição de frequência, segundo os valores médios de qualidade de vida: "ruim" (de 1,0 até 2,9); "regular" (de 3,0 a 3,9) e "boa" (acima de 4,0).

Todos os dados obtidos nas coletas foram tabulados e analisados, no software Statistical Package for the Social Sciences® (SPSS), versão 21. Foram conduzidas análises descritivas e de medidas de frequências (absoluta e relativa) e tendência central (média).

O tempo de cirurgia foi dividido em quartis ficando da seguinte forma: no $1^{\circ}$ quartil $(0$ a 444 dias $) 77$ pacientes; para o $2^{\circ}$ quartil (445 até 1165 dias) 76 pacientes; $3^{\circ}$ quartil (1166 até 2249 dias) 76 pacientes e $4^{\circ}$ quartil (2250 até 4952 dias) 76 pacientes. Essa divisão foi feita baseada em uma análise estatística para propiciar maior fidedignidade ao estudo.

Os dados coletados, para a realização desta pesquisa, obedeceram a todas as normas e salvaguardas estabelecidas pelo Comitê de Ética em Pesquisa (CEP) da Universidade Federal de São João Del-Rei, Campus Centro-Oeste Dona Lindu (UFSJ/CCO). Todo o processo seguiu os termos da Resolução n. ${ }^{\circ} 466$ de 12 de dezembro de 2012, do Conselho Nacional de Ética em Pesquisa (CONEP), que trata de normas sobre a pesquisa envolvendo seres humanos. O estudo foi aprovado sob o parecer de $\mathrm{N}^{\mathrm{o}} 3.330 .917$.

\section{Resultados}

Neste estudo, foram avaliados 305 pacientes que foram submetidos à cirurgia bariátrica. A caracterização sociodemográfica mostrou predominância de pacientes do sexo feminino (255-83,6\%), da faixa etária entre 31 e 40 anos (127$41,6 \%$ ), casados (188-61,6\%), com ensino superior completo (128-42\%) e com renda familiar mensal superior a três salários mínimos $(148-48,5 \%)$.

As Tabelas 1, 2 e 3, apresentadas a seguir, representam a distribuição do $n=305$ divididos em quartis, no que se refere à acurácia perceptiva, satisfação com a imagem corporal e qualidade de vida por meio da divisão entre os domínios (físico, psicológico, meio ambiente, qualidade de vida geral).

A divisão do tempo se deu pela distância interquartílica, ou seja, foi dividido o tempo em quatro partes de forma que o número de pacientes ficasse igual em cada faixa de tempo. Para cada tabela, foram gerados dois $\mathrm{P} *$ valor divididos entre homens e mulheres, para testar linha e coluna, sendo as linhas representando as variáveis e a coluna representando seus respectivos quartis para testar a associação entre as variáveis.

A Tabela 1 apresenta a distribuição dos pacientes segundo a classificação da acurácia perceptiva da imagem corporal por sexo e sua associação com o tempo de cirurgia bariátrica. 
Tabela 1 - Distribuição dos pacientes segundo a classificação da acurácia perceptiva da imagem corporal da escala de Figuras de Silhuetas e o tempo de cirurgia bariátrica. Divinópolis, MG, Brasil, 2020.

\begin{tabular}{|c|c|c|c|c|c|c|c|c|c|c|}
\hline \multicolumn{11}{|c|}{ Acurácia Perceptiva da Imagem Corporal x Tempo de Cirurgia Bariátrica } \\
\hline \multirow[b]{2}{*}{$\begin{array}{c}\text { Acurácia } \\
\text { Perceptiva }\end{array}$} & \multicolumn{5}{|c|}{ Homens } & \multicolumn{5}{|c|}{ Mulheres } \\
\hline & $1^{\circ}$ quartil & $2^{\circ}$ quartil & $\begin{array}{c}\mathbf{3}^{\circ} \\
\text { quartil }\end{array}$ & $\begin{array}{c}4^{\circ} \\
\text { quartil }\end{array}$ & Valor-p* & $\mathbf{1}^{\circ}$ quartil & $2^{\circ}$ quartil & $3^{\circ}$ quartil & $\begin{array}{c}4^{\mathbf{o}} \\
\text { quartil }\end{array}$ & Valor-p* \\
\hline Superestima & 5 & 6 & 9 & 10 & \multirow{4}{*}{0,802} & 44 & 35 & 40 & 44 & \multirow{4}{*}{0,123} \\
\hline Acurácia & 4 & 2 & 4 & 3 & & 13 & 25 & 17 & 14 & \\
\hline Subestima & 1 & 3 & 1 & 2 & & 10 & 5 & 5 & 3 & \\
\hline Total & 10 & 11 & 14 & 15 & & 67 & 65 & 62 & 61 & \\
\hline
\end{tabular}

*Teste qui-quadrado de Pearson (Nível de significância p<0,05).

Fonte: Dados da pesquisa (2020).

A Tabela 2 apresenta a distribuição dos pacientes segundo a classificação da satisfação com a imagem corporal por sexo e sua associação com o tempo de cirurgia bariátrica.

Tabela 2 - Distribuição dos pacientes segundo a classificação da satisfação com a imagem corporal da escala de Figuras de Silhuetas e o tempo de cirurgia bariátrica, Divinópolis, MG, Brasil, 2020.

\begin{tabular}{|c|c|c|c|c|c|c|c|c|c|c|}
\hline \multicolumn{11}{|c|}{ Satisfação com a Imagem Corporal x Tempo de Cirurgia Bariátrica } \\
\hline \multirow{2}{*}{$\begin{array}{c}\text { Satisfação } \\
\text { Imagem Corporal }\end{array}$} & \multicolumn{5}{|c|}{ Homens } & \multicolumn{5}{|c|}{ Mulheres } \\
\hline & $1^{\circ}$ quartil & $\begin{array}{c}2^{\circ} \\
\text { quartil } \\
\end{array}$ & $\begin{array}{c}3^{\circ} \\
\text { quartil } \\
\end{array}$ & $4^{\circ}$ quartil & Valor-p* & $\begin{array}{c}1^{\circ} \\
\text { quartil } \\
\end{array}$ & $2^{\circ}$ quartil & $\begin{array}{c}3^{\circ} \\
\text { quartil } \\
\end{array}$ & $\begin{array}{c}4^{\circ} \\
\text { quartil } \\
\end{array}$ & Valor-p* \\
\hline $\begin{array}{c}\text { Insatisfeito } \\
\text { Perdeu pouco peso }\end{array}$ & 6 & 9 & 12 & 10 & \multirow{4}{*}{0,284} & 51 & 40 & 45 & 46 & \multirow{4}{*}{0,456} \\
\hline Satisfeito & 0 & 1 & 0 & 0 & & 13 & 23 & 15 & 14 & \\
\hline $\begin{array}{c}\text { Insatisfeito } \\
\text { Perdeu muito peso }\end{array}$ & 4 & 1 & 2 & 5 & & 3 & 2 & 2 & 1 & \\
\hline Total & 10 & 11 & 14 & 15 & & 67 & 65 & 62 & 61 & \\
\hline
\end{tabular}

*Teste qui-quadrado de Pearson (Nível de significância $p<0,05$ ).

Fonte: Dados da pesquisa (2020).

A Tabela 3 apresenta a distribuição dos pacientes segundo a classificação da qualidade de vida por sexo e sua associação com o tempo de cirurgia bariátrica. 
Tabela 3 - Distribuição dos pacientes segundo a classificação da qualidade de vida por domínios e o tempo de cirurgia bariátrica. Divinópolis, MG, Brasil, 2020.

\begin{tabular}{|c|c|c|c|c|c|c|c|c|c|c|c|}
\hline \multicolumn{12}{|c|}{ Qualidade de Vida por Domínios x Tempo de Cirurgia Bariátrica } \\
\hline \multirow{2}{*}{\multicolumn{2}{|c|}{$\begin{array}{c}\text { Qualidade } \\
\text { de Vida por Domínios }\end{array}$}} & \multicolumn{5}{|c|}{ Homens } & \multicolumn{5}{|c|}{ Mulheres } \\
\hline & & \multirow{2}{*}{$\begin{array}{c}\mathbf{1}^{\mathbf{0}} \\
\text { quartil } \\
8\end{array}$} & \multirow{2}{*}{\begin{tabular}{|c|}
$\begin{array}{c}\mathbf{2}^{\mathbf{0}} \\
\text { quartil }\end{array}$ \\
6 \\
\end{tabular}} & \multirow{2}{*}{\begin{tabular}{|c|}
$3^{\mathbf{o}}$ \\
quartil \\
9
\end{tabular}} & \multirow{2}{*}{$\begin{array}{c}\begin{array}{c}4^{\mathbf{0}} \\
\text { quartil }\end{array} \\
11\end{array}$} & $\begin{array}{c}\text { Valor } \\
\text { p* }\end{array}$ & $\begin{array}{c}1^{\circ} \\
\text { quartil }\end{array}$ & $\begin{array}{c}2^{\circ} \\
\text { quartil }\end{array}$ & \begin{tabular}{|c|}
$3^{\mathbf{o}}$ \\
quartil
\end{tabular} & \begin{tabular}{c|}
$4^{\circ}$ \\
quartil
\end{tabular} & $\begin{array}{c}\text { Valor- } \\
\text { p* }\end{array}$ \\
\hline \multirow[b]{3}{*}{ Físico } & Boa $(>4,0)$ & & & & & \multirow{3}{*}{0,916} & 36 & 42 & 42 & 37 & \multirow{3}{*}{0,529} \\
\hline & $\begin{array}{c}\text { Regular }(3,0 \\
\text { a 3,9) }\end{array}$ & 2 & 4 & 4 & 3 & & 21 & 18 & 17 & 17 & \\
\hline & \begin{tabular}{|c|} 
Ruim (até \\
$2,9)$
\end{tabular} & 0 & 1 & 1 & 1 & & 10 & 5 & 3 & 7 & \\
\hline \multirow{3}{*}{ Psicológico } & Boa $(>4,0)$ & 8 & 5 & 7 & 9 & \multirow{3}{*}{0,197} & 38 & 39 & 40 & 33 & \multirow{3}{*}{0,758} \\
\hline & $\begin{array}{c}\text { Regular }(3,0 \\
\text { a } 3,9)\end{array}$ & 2 & 4 & 3 & 6 & & 23 & 19 & 15 & 18 & \\
\hline & \begin{tabular}{|c|} 
Ruim (até \\
$2,9)$
\end{tabular} & 0 & 2 & 4 & 0 & & 6 & 7 & 7 & 10 & \\
\hline \multirow{3}{*}{$\begin{array}{l}\text { Relações } \\
\text { Sociais }\end{array}$} & Boa $(\geq 4,0)$ & 6 & 6 & 8 & 12 & \multirow{3}{*}{0,462} & 41 & 45 & 38 & 31 & \multirow{3}{*}{0,184} \\
\hline & $\begin{array}{c}\text { Regular }(3,0 \\
\text { a } 3,9)\end{array}$ & 4 & 4 & 3 & 2 & & 16 & 14 & 21 & 22 & \\
\hline & \begin{tabular}{|c|} 
Ruim (até \\
$2,9)$
\end{tabular} & 0 & 1 & 3 & 1 & & 10 & 6 & 3 & 8 & \\
\hline \multirow{3}{*}{ Meio Ambiente } & Boa $(\geq 4,0)$ & 7 & 5 & 5 & 8 & \multirow{3}{*}{0,419} & 31 & 37 & 34 & 21 & \multirow{3}{*}{0,225} \\
\hline & $\begin{array}{c}\text { Regular }(3,0 \\
\text { a } 3,9)\end{array}$ & 3 & 5 & 6 & 7 & & 28 & 23 & 23 & 33 & \\
\hline & \begin{tabular}{|c|} 
Ruim (até \\
$2,9)$ \\
\end{tabular} & 0 & 1 & 3 & 0 & & 8 & 5 & 5 & 7 & \\
\hline \multirow{3}{*}{$\begin{array}{l}\text { Qualidade de } \\
\text { Vida Geral }\end{array}$} & Boa $(\geq 4,0)$ & 8 & 8 & 7 & 12 & \multirow{3}{*}{0,452} & 51 & 54 & 52 & 41 & \multirow[b]{3}{*}{0,253} \\
\hline & $\begin{array}{c}\text { Regular }(3,0 \\
\text { a } 3,9)\end{array}$ & 2 & 1 & 5 & 2 & & 12 & 10 & 7 & 16 & \\
\hline & \begin{tabular}{|c|} 
Ruim (até \\
$2,9)$
\end{tabular} & 0 & 2 & 2 & 1 & & 4 & 1 & 3 & 4 & \\
\hline
\end{tabular}

*Teste qui-quadrado de Pearson (Nível de significância p<0,05).

Fonte: Dados da pesquisa (2020).

\section{Discussão}

Apesar de não ter sido encontrada uma diferença estatisticamente significante entre os sexos, com relação à acurácia perceptiva da imagem corporal, chama atenção o fato de que, proporcionalmente, as mulheres foram as que mais realizaram cirurgia bariátrica (83,6\%). Esse fato pode estar associado à grande pressão social e midiática por um corpo magro principalmente direcionado às mulheres (Lima, Brito, Tomiya, Souza, \& Silva, 2018).

Porém não apenas a mídia impõe tal modelo às mulheres e homens, a própria visão biomédica mostra que o corpo magro é o desejável e o saudável. Tal evento pode ser explicado pelo fato de que a cultura ocidental hipervalorizar a dimensão biológica dos corpos e processos corporais. Nesse sentido, essa visão acaba incitando a população pela busca constante à modificação do corpo, por meio de intervenções estéticas, colocando grande pressão nos indivíduos que não se encaixam nos padrões impostos, levando-os até mesmo a enfrentar transtornos emocionas que podem, por exemplo, agravar o consumo de alimentos (Separavich \& Canesqui, 2010). Outro fator pode estar ligado ao atual contexto social, que é uma hipervalorização 
dos corpos veiculados na mídia, sendo o corpo magro idealizado, sobretudo, pelas mulheres (De Carvalho, Gomes, \& Ferreira, 2016).

Neste sentido, destaca-se a importância de práticas educativas a serem realizadas pelo profissional de saúde em prol dessa população. É crucial que o paciente seja orientado acerca dos benefícios e malefícios da cirurgia e obtenha auxílio na tomada de decisão, sobretudo, no direcionamento das suas escolhas e da real motivação de se realizar tal procedimento.

Apesar de ter se apresentado em menor proporção, quando comparado às mulheres, destaca-se também o aumento por procedimentos cirúrgicos realizados por homens. A ideologia de um corpo portador de medidas ideais, como resultado do imaginário coletivo permeado pela cultura narcisista, pode levar à busca do sucesso individual materializado no corpo (Leal, Catrib, Amorim, \& Montagner, 2010). Sendo assim, ao falar de imaginário coletivo e cultura narcisista, infere-se que tanto os homens quanto as mulheres estão implicados nesse processo.

Quanto ao estado civil, 61,6\% dos pacientes eram casados. Não há muitos estudos que relacionam o estado civil com a CB. Porém há relatos de que pacientes que têm parceiros apresentam maior desejo de perda de peso, em decorrência de alguma exigência do casamento, como a não satisfação do parceiro (Lima et al., 2018).

Nessa ótica, cabe evidenciar que a decisão de realizar um tratamento cirúrgico para o casal pode ser tida como um reavivamento do relacionamento, uma forma de reafirmação do desejo de fazer o melhor para o outro (Arantes, 2008). Entretanto o desejo pode ser uma via de acolhimento de um ideal e percebe-se que o corpo/beleza é ditado como algo que pode ser comprado (Tinoco \& Sirelli, 2014).

Sendo assim, a normatização da imagem corporal parece passar pelo aspecto ligado ao ideal de beleza e, por conseguinte, pela apreciação do outro. Um estudo realizado por Domínguez (2011) aborda a questão do corpo como algo que pode ser apreciado apenas como uma imagem. O "corpo imagem" se constitui, também, como fundamento da autoestima, sendo ela atualmente reduzida a questões de ordem subjetiva, moldada em uma cultura narcisista, na qual a imagem que se oferece ao mundo passa pela necessidade de aceitação social (Leal et al, 2010).

No que se refere à faixa etária (41,6\% - entre 31 e 40 anos), escolaridade (ensino superior completo - 42\%) e renda familiar (48,5\% - superior a três salários mínimos), tem-se, com a idade, um ganho de peso esperado, sendo que da adolescência para a idade adulta o indivíduo ganha um aumento em seu IMC.

A CB no Brasil não é permitida, antes dos 16 anos de idade e, mesmo nessa faixa etária, é necessário um acompanhamento multiprofissional, para se tomar a decisão de realizar essa cirurgia. No país não se têm muitos estudos sobre CB na adolescência, o que pode levar muitos profissionais da saúde a não aconselharem esse procedimento (Marcelino \& Patrício, 2011; Massabki et al., 2016), assim, seguem tratamentos mais conservadores que podem não dar resultados imediatos ou não conseguirem sozinhos resolver a problemática, até que, ao se adentrar na idade adulta, com as demandas sociais e comorbidades secundárias, o indivíduo assuma a responsabilidade pela CB.

Estudo realizado por Neto, Oliveira e Silva (2018) concorda com os resultados da presente pesquisa quanto à renda familiar, os quais mostram que uma renda familiar adequada se relaciona à maior aptidão ao trabalho e maior envolvimento com atividades físicas pós-CB e maior tendência se conseguir uma redução do IMC.

Quanto à escolaridade, o estudo está de acordo com os resultados deJunge et al. (2016), em que a maior parcela tem ensino médio completo e superior, mas outros estudos discordam quanto à importância da escolaridade (Eduardo, Silva, Cruz, Viana, \& Rocha, 2017), podendo estar relacionada ao perfil dos participantes que procuram uma clínica particular para realizar o procedimento. O perfil dos pacientes que procura uma clínica particular pode também estar associado ao nível da renda familiar demonstrada neste estudo, superior a três salários mínimos. 
O estudo demonstrou que houve distorção da imagem corporal independentemente do sexo em todos os quartis. A distorção da imagem pode ser associada a medos de retorno ao peso anterior, insatisfação com a imagem atual e a insegurança com o corpo atual (Eduardo et al., 2017).

Os resultados também demonstraram que a maioria dos pacientes superestimam sua imagem corporal, ou seja, escolheram a figura mais obesa representando seu corpo atual e uma figura mais magra para a figura que representa o corpo que acreditam ter após a cirurgia bariátrica, também nos 4 quartis. Esse resultado relaciona-se com outros estudos sobre a temática (Segura, Corral, Wozniak, Scaravonatto, Vandresen, 2016; Lacerda et al., 2018).

Ao se realizar uma $\mathrm{CB}$, muitos pacientes acreditam que irão ter a solução dos seus problemas e, ao perceberem que com a cirurgia advêm outras dificuldades, como a diminuição da absorção de nutrientes, perda muito rápida de peso com perda da elasticidade da pele e até mesmo ganho de peso com o passar dos anos, esses indivíduos podem desenvolver desvios da imagem corporal (Lacerda et al., 2018).

Estudo conduzido por Lacerda et al. (2018) analisou a percepção da imagem corporal de pacientes submetidos à CB, demonstrando que também tinham distorção da imagem, sendo que a insatisfação era maior entre os indivíduos que realizaram a CB há pelo menos seis meses. A maior distorção da imagem foi encontrada no grupo de pacientes com 12 a 24 meses pósoperatório. Já o presente estudo não demonstrou variação, dentro do tempo estudado, para as variáveis distorção da imagem e satisfação com a imagem corporal.

A variável qualidade de vida também não demonstrou alterações estatisticamente significativas nos quartis nos quatro domínios: físico, psicológico, relações sociais e meio ambiente. A maior frequência, de acordo com o questionário Whoqol bref, foi considerada "boa". Contudo, a partir desses achados, é relevante maior reflexão quanto ao domínio psicológico, uma vez que a distorção da imagem corporal pode ter implicações no aspecto psíquico.

No entanto cabe destacar que a qualidade de vida geral demonstrou um resultado satisfatório, de maneira geral. Esse achado demonstra que a CB parece ser eficaz ao tratamento da obesidade, trazendo para os indivíduos melhora na qualidade de vida, apesar dos medos e possíveis frustrações de não se ter o corpo perfeito. Estudos demonstram que com a CB têm-se melhora do humor, melhora da autoestima e do interesse sexual (Eduardo et al., 2017; Marchesini \& Antunes, 2019).

Percebe-se, portanto que a efetividade do respectivo procedimento não está associada apenas à perda de peso, uma vez que estudos apontam sua relevância na reeducação alimentar e estilo de vida mais saudáveis. Isso pode ser evidenciado por outros estudos, indicando que o procedimento cirúrgico em questão pode impactar positivamente na saúde física e mental dos pacientes (Castanha et al., 2018).

Portanto, apesar de, culturalmente, a CB estar inserida em um contexto que, de forma ou de outra, contribui com o culto à estética corporal, tal prática pode repercutir positivamente e de forma individual na qualidade de vida dos pacientes. Faz-se necessário o acompanhamento periódico por profissionais da saúde dos indivíduos que passaram por esse procedimento para que sejam identificadas possíveis alterações na qualidade de vida (Oliveira et al., 2018). Tal assertiva vai ao encontro do proposto e achado nesta pesquisa, uma vez que os pacientes foram avaliados, em um período de até 15 anos após a cirurgia e observa-se, na literatura, uma limitação quanto a esse tempo.

Outras pesquisas também demonstraram a efetividade da CB para a qualidade de vida. Como o estudo de Duarte et al. (2014) que avaliou 57 indivíduos submetidos ao procedimento, após 12 a 36 meses da cirurgia e os compararam com um grupo que aguardava para a realização da CB. O resultado demonstrou que os que se submeterem ao procedimento tiverem resultados mais satisfatórios quanto à qualidade de vida que o grupo não submetido.

Importante ressaltar que os estudos utilizados para a discussão não pesquisaram indivíduos submetidos à $\mathrm{CB}$, dentro do tempo de pós-cirurgia, conforme o estudo aqui apresentado, razão pela qual seria de vital importância a realização de outras pesquisas, com cenários similares para se discutir melhor com este estudo. 
Infere-se que a qualidade de vida se relaciona com a CB de forma positiva, mas a autoimagem subjetiva deve ser considerada fundamental, como demonstrado na pesquisa, pois uma autoimagem negativa pode trazer consequências, em longo prazo negativas, como depressão, transtornos alimentares com consequente ganho de peso ou sua perda excessiva levando assim a um forte impacto sobre a qualidade de vida (Lacerda et al., 2018).

Conforme Tonatto-Filho, Gallotti, Chedid, Grezzana-Filho e Garcia (2019), comparando-se os resultados da cirurgia bariátrica com o tratamento clínico, principalmente no que diz respeito às comorbidades ligadas à obesidade, o tratamento cirúrgico é mais eficaz. Há de se considerar que, no Brasil, mais de um milhão de pessoas estão com obesidade grave. Pode-se afirmar que os achados deste trabalho têm impacto significativo, no âmbito da saúde, uma vez que elucida alguns aspectos ligados à complexidade acerca da cirurgia bariátrica, munindo assim não só os profissionais ligados a esse procedimento quanto os pacientes no que tange à sua saúde e a suas expectativas.

\section{Considerações Finais}

Este estudo procurou avaliar a acurácia perceptiva, a satisfação com a imagem corporal e a qualidade de vida dos pacientes bariatrizados, bem como a associação com o tempo pós-cirurgia bariátrica.

A presente pesquisa demonstrou, por meio de divisão do tempo usando a distância interquartílica, que há distorção e insatisfação dos pacientes quanto à sua imagem corporal no período estudado. A qualidade de vida foi considerada boa em todos os quartis. É importante salientar que não houve influência do tempo de cirurgia quanto a esse resultado.

Ainda, destaca-se que estudos sobre qualidade de vida carecem de uma definição mais precisa quanto ao tempo e período de sua avaliação, após o procedimento, uma vez que estudos científicos não convergem ao mesmo ponto quanto a essa questão. Sendo assim, percebe-se a necessidade de mais pesquisas nessa perspectiva. Outro fator que merece observação é a escassez de pesquisas nacionais e internacionais mais atuais relacionadas à temática desta pesquisa que leve em consideração um tempo maior de pós-cirurgia bariátrica.

Desta forma, torna-se relevante novos estudos que objetivem a avaliação a longo prazo de pacientes que fizeram cirurgia bariátrica. Da mesma maneira, é necessário que outros trabalhos investiguem a compreensão dos períodos em que estes pacientes devem ser submetidos à avaliação que contemplem as variáveis levantadas nesta pesquisa para agregar maior efetividade a esse procedimento.

\section{Referências}

Andrade, J. R. D., Velasquez-Melendez, G., Barreto, S. M., Pereira, T. S. S., Mill, J. G., \& Molina, M. D. C. B. (2017). Fenótipo da cintura hipertrigliceridêmica e fatores nutricionais: um estudo com participantes do ELSA-Brasil. Revista Brasileira de Epidemiologia, 20 (3), $382-393$.

Arantes, F. B. (2008). O Impacto da Cirurgia Bariátrica na Relação de Casal: Uma Perspectiva dos Cônjuges Masculinos (Dissertação de Mestrado). Pontifícia Universidade Católica de Goiás, Goiânia, GO.

Brasil. (2019). Ministério da Saúde. Secretaria de Vigilância em Saúde. Departamento de Análise em Saúde e Vigilância de Doenças não Transmissíveis. Vigitel Brasil 2018: vigilância de fatores de risco e proteção para doenças crônicas por inquérito telefônico: estimativas sobre frequência e distribuição sociodemográfica de fatores de risco e proteção para doenças crônicas nas capitais dos 26 estados brasileiros e no Distrito Federal em 2018. Brasília: Ministério da Saúde.

Castanha, C. R., Ferraz, A. A. B., Castanha, A. R., Belo, G. Q. M. B., Lacerda, R. M. R., \& Vilar, L. (2018). Avaliação da qualidade de vida, perda de peso e comorbidades de pacientes submetidos à cirurgia bariátrica. Revista do Colégio Brasileiro de Cirurgiões, 45(3), e1864.

Castro, I. R. R. (2017). Obesity prevention and control: the urgent need for effective public policies. Cadernos de Saúde Pública, 33 (7), e00100017.

De Carvalho, P. H. B., Gomes, L. de F., \& Ferreira, M. E. C. (2016). Comparação social, insatisfação corporal e comportamento alimentar em jovens adultos. Interação em Psicologia, 20(2), 219-225.

Domínguez, H. T. (2011). El cuerpo en la experiencia analítica. Freudiana, 63, 29-32. 
Duarte, M. I. X. de T., Bassitt, D. P., Azevedo, O. C., Waisberg, J., Yamaguchi, P., \& Pinto Junior, P. E. (2014). Impact on quality of life, weight loss and comorbidities: a study comparing the biliopancreatic diversion with duodenal switch and the banded Roux-en-Y gastric bypass. Arquivos de Gastroenterologia, 51(4), 320-327.

Estrela, C. (2018). Metodologia Científica: Ciência, Ensino, Pesquisa. Editora Artes Médicas.

Eduardo, C. A., Silva, A. A., Cruz, C. D., Viana, G. C. P., \& Rocha, R. L. P. (2017). Cirurgia bariátrica: a percepção do paciente frente ao impacto físico, psicológico e social. Revista de Enfermagem do Centro Oeste Mineiro, 7, e1173.

Flegal, K., Kit, B. K., \& Graubard, B. I. (2014). Body mass index categories in observational studies of weight and risk of death. American Journal of Epidemiology, 180(3), 288-296.

Francisco, L., \& Diez-Garcia, R. W. (2015). Abordagem terapêutica da obesidade: entre conceitos e preconceitos. DEMETRA: Alimentação, Nutrição \& Saúde, 10, 705-716.

Junges, A. H., Bremm, C., Fontana, D. C., Oliveira, C. A. O. D., Schaparini, L. P. \& Carvalho, P. C. D. F. (2016). Temporal profiles of vegetation indices for characterizing grazing intensity on natural grasslands in Pampa biome. Scientia Agricola, 73, 332-337.

Kakeshita, I. S., \& Almeida, S. D. S. (2006). Relação entre índice de massa corporal e a percepção da auto-imagem em universitários. Revista de Saúde Pública, 40, 497-504.

Lacerda, R. M. R., Castanha, C. R., Castanha, A. R., Campos, J. M., Ferraz, A. A. B., \& Vilar, P. (2018). Percepção da imagem corporal em pacientes submetidos à cirurgia bariátrica. Revista do Colégio Brasileiro de Cirurgiões, 45(2), e1793.

Leal, V. C. L. V., Catrib, A. M. F., Amorim, R. F., \& Montagner, M. A. (2010). O corpo, a cirurgia estética e a Saúde Coletiva: um estudo de caso. Ciência e Saúde Coletiva, 15(1), 77-86.

Lima, A. R., Brito, L. S., Tomiya, M. T. O., Souza, T. K. M., \& Silva, R. P. P. (2018). Evolução ponderal e qualidade de vida de indivíduos submetidos à cirurgia bariátrica. Revista Brasileira de Obesidade, Nutrição e Emagrecimento, 12(76), 1087-1094.

Malta, D., Moura, L., Prado, R. R., Escalante, J. C., Schmidt, M. I., \& Duncan, B. B. (2014). Mortalidade por doenças crônicas não transmissíveis no Brasil e suas regiões, 2000 a 2011. Epidemiologia e Serviços de Saúde, 23, 599-608.

Marcelino, L. F., \& Patricio, Z. M. (2011). A complexidade da obesidade e o processo de viver após a cirurgia bariátrica: uma questão de saúde coletiva. Ciência e Saúde Coletiva, 16(12), 4767-4776.

Marchesini, S. D., \& Antunes, M. C. (2017). A percepção do corpo em pacientes bariátricos e a experiência do medo do reganho do peso. Interação em Psicologia, 21(2), 127-136

Massabki, L. H. P., Sewaybricker, L. E., Nakamura, K. H., Mendes, R. T., Barros Filho, A. A., Antonio, M. A. R. G. M. ... Zambon, M. P. (2016). Cirurgia bariátrica: é razoável antes dos 16 anos de idade? Revista do Colégio Brasileiro de Cirurgia, 43(5), 360-367.

Neto, P. P., Oliveira, A. A. R. de., \& Silva, C. A. B, da. (2018). Avaliação da qualidade de vida associada à obesidade em indivíduos submetidos à cirurgia bariátrica. Motricidade, 14(1), 123-132.

Oliveira, L. S. F. de., Mazini Filho, M. L., Castro, J. B. P., Touguinha, H. M., Silva, P. C. R., \& Ferreira, M. E. C. (2018). Repercussões da cirurgia bariátrica na qualidade de vida, no perfil bioquímico e na pressão arterial de pacientes com obesidade mórbida. Fisioterapia e Pesquisa, 25 (3), $284-293$.

OMS - World Health Organization. 2015. Obesity and overweight. Recuperado de https://www.who.int/news-room/fact-sheets/detail/obesity-and-overweight.

Segura, D. C. A., Corral, J. P., Wozniak, S. D., Scaravonatto, A., \& Vandresen, E. P. (2016). Análise da imagem corporal e satisfação com o peso em indivíduos submetidos à cirurgia bariátrica. Revista Pesquisa e Saúde, 17(3), 170-174.

Separavich, M. A., \& Canesqui, A. M. (2010). Girando a lente socioantropológica sobre o corpo: uma breve reflexão. Saude \& Sociedade, 19(2), $249-259$.

Steyer, N., Oliveira, M. C., Gouvêa, M. R. F., Echer, I. C., \& Lucena, A. F. (2016). Perfil clínico, diagnósticos e cuidados de enfermagem para pacientes em pós-operatório de cirurgia bariátrica. Revista Gaúcha de Enfermagem, 37, e50170.

Tinoco, D. L., \& Sirelli, N. M. O corpo a serviço de um ideal: subjetividade e cultura. Psicanálise \& Barroco em revista, 12(1), 148-171.

Tonatto-Filho, A. J., Gallotti, F. M., Chedid, M. F., Grezzana-Filho, T. J. M., \& Garcia, A. M. S. V. (2019). Bariatric surgery in Brazilian public health system: The goog, The bad and the ugly, or a long way to go, yellow sing!. Arquivos Brasileiros de Cirurgia Digestiva, 32(4), e1470.

Wu, C. Y., Chou, Y. C., Huang, N., Chou, Y. J., Hu, H. Y., \& Li, C. P. (2014). Association of body mass index with all-cause and cardiovascular disease mortality in the elderly. PLoS One, 9 (7), e102589. 